\title{
Automata Slicing for Diagnosing Discrete-Event Systems with Partially Ordered Observations
}

\author{
Alban Grastien ${ }^{1}$, Marie-Odile Cordier ${ }^{1}$, and Christine Largouët ${ }^{2}$ \\ 1 Irisa, University of Rennes 1, Campus de Beaulieu, 35042 Rennes Cedex, France \\ \{agrastie, cordier\}@irisa.fr \\ 2 University of New Caledonia, BP. 4477, 98847 Nouméa Cedex, New Caledonia \\ largouet@univ-nc.nc
}

\begin{abstract}
When dealing with real systems, it is unrealistic to suppose that observations can be totally ordered according to their emission dates. The partially ordered observations and the system are thus both represented as finite-state machines (or automata) and the diagnosis formally defined as the synchronized composition of the model with the observations. The problem we deal with in this paper is that, taking into account partially ordered observations rather than sequential ones, it becomes difficult to consider the observations one after the other and to incrementally compute the global diagnosis.

In this paper, we rely on a slicing of the observation automata and propose to compute diagnosis slices (for each observation slice) before combining them to get the global diagnosis. In order to reach this objective, we introduce the concept of automata chain and define the computation of the diagnosis using this chain, first in a modular way and then, more efficiently, in an incremental way. These results are then extended to the case where observations are sliced according to temporal windows. This study is done in an off-line context. It is a first and necessary step before considering the on-line context which is discussed in the conclusion.
\end{abstract}

\section{Introduction}

It is established that diagnosing dynamical systems, represented as discrete-event systems [1] amounts to finding what happened to the system from existing observations $[2,3,4,5,6]$. In this context, the diagnostic task consists in determining the trajectories (a sequence of states and events) compatible with the observations. When dealing with real systems, it is unrealistic to suppose that observations can be totally ordered according to their emission dates. The partially ordered observations and the system are thus both represented as finite-state machines (or automata) and the diagnosis formally defined as the synchronized composition of the model with the observations.

A problem that can be encountered is the size of the observation automaton, due to the temporal uncertainties on the observations or/and the duration of the observation recording. For instance, we may want to compute an a posteriori diagnosis from log files of observations during a few days period, as in the domain of telecommunication networks. It becomes difficult to consider the observations 
one after the other and to incrementally compute the global diagnosis. In this article, we propose a way to avoid this global computation by considering an automata slicing for the observations and building the diagnosis incrementally on successive slices of observations. The problem of building the sliced observation automata is not considered in this paper where we consider it as given.

After a brief reminder of the definitions about automata (section 2), we introduce, in section 3 , the concept of automata chain, to represent an automaton by a sequence of automata slices. We provide the properties such an automata chain has to satisfy to be a correct slicing and define a reconstruction operation to get the global automaton back. Then, we demonstrate, provided the observations are correctly sliced, that the diagnosis can be correctly (section 4) and incrementally (section 5) computed from the observation slices. In section 6, these results are extended to the case where observations are sliced according to time, i.e according to temporal windows. We here focus on the off-line diagnosis context; the extension to the on-line diagnosis context is discussed in the conclusion.

\section{Preliminaries: automata and trajectories}

In this paper, we are more particularly interested in diagnosing reactive systems. Reactive systems are event-driven since their behaviour evolves with the occurrence of events and can cause by propagation a succession of state changes [2]. In this approach, the behavioural model of the system is represented by finite state machines. This section thus recalls some basic notions about automata and trajectories.

Definition 1 (Automaton) An automaton $A$ is a tuple $(Q, E, T, I, F)$ where:

$-Q$ is the finite set of states;

$-E$ is the finite set of events;

$-T \subseteq\left(Q \times 2^{E} \times Q\right)$ is the finite set of transitions; a transition $t$ is a tuple $\left(q, \bar{l}, q^{\prime}\right)$ such that $t$ connects $q$ to $q^{\prime}$ on the label $l$, with $l \in 2^{E} \backslash\{\emptyset\}$ a non-empty subset of events;

- I is the finite set of initial states $(I \subseteq Q)$; and

$-F$ is the finite set of final states $(F \subseteq Q)$.

Labels over transitions should not be empty. We consider that $\forall q \in Q$, the transition $(q, \emptyset, q)$ is a transition of $T$.

A path between the states $q_{0}$ and $q_{m}$ of an automaton $A=(Q, E, T, I, F)$ is the couple $\left(\left(q_{0}, \ldots, q_{m}\right),\left(l_{1}, \ldots, l_{m}\right)\right)$, where $\left(q_{0}, \ldots, q_{m}\right)$ is the finite sequence of states and $\left(l_{1}, \ldots, l_{m}\right)$ the sequence of labels, such that:

- $\forall i \in\{0, \ldots, m\}, q_{i} \in Q$, and

$-\forall i \in\{1, \ldots, m\}, t_{i}=\left(q_{i-1}, l_{i}, q_{i}\right) \in T$.

A trajectory denoted traj of an automaton $A$ is a path $\left(\left(q_{0}, \ldots, q_{m}\right),\left(l_{1}, \ldots, l_{m}\right)\right)$, where $q_{0} \in I$ and $q_{m} \in F$.

Two automata $A$ and $A^{\prime}$ are equal $\left(A=A^{\prime}\right)$ if their trajectory sets are equal. We call simplified automaton of $A$ the automaton $A^{\prime}=A$ where all the 
states and transitions that do not appear in at least one trajectory have been removed. In the following, when computing new automata, only simplified ones are considered.

Definition 2 (Synchronization of labels) Given $l_{1}$ a label from $E_{1}$ and $l_{2}$ a label from $E_{2}, l_{1}$ and $l_{2}$ are said to be synchronized iff $l_{1} \cap\left(E_{1} \cap E_{2}\right)=$ $l_{2} \cap\left(E_{1} \cap E_{2}\right)$. The synchronization $l$, denoted $\Theta\left(l_{1}, l_{2}\right)$, is the label $l_{1} \cup l_{2}$ on the set of events $E_{1} \cup E_{2}$.

Two labels can be synchronized if the synchronization events $\left(E_{1} \cap E_{2}\right)$ are common to both labels. Note that $l_{1}=l \cap E_{1}$ and $l_{2}=l \cap E_{2}$.

Definition 3 (Synchronization of automata) Let $A_{1}=\left(Q_{1}, E_{1}, T_{1}, I_{1}, F_{1}\right)$ and $A_{2}=\left(Q_{2}, E_{2}, T_{2}, I_{2}, F_{2}\right)$ be two automata. The synchronization of $A_{1}$ and $A_{2}$, denoted $A_{1} \otimes A_{2}$, is the automaton $A=(Q, E, T, I, F)$ such that:

$-Q=Q_{1} \times Q_{2}$

$-E=E_{1} \cup E_{2}$,

$-T=\left\{\left(\left(q_{1}, q_{2}\right), l,\left(q_{1}^{\prime}, q_{2}^{\prime}\right)\right) \mid\left(q_{1}, l \cap E_{1}, q_{1}^{\prime}\right) \in T_{1} \wedge\left(q_{2}, l \cap E_{2}, q_{2}^{\prime}\right) \in T_{2}\right\}$,

$-I=I_{1} \times I_{2}$, and

$-F=F_{1} \times F_{2}$.

The synchronization consists in trigerring simultaneously the two transitions having the same synchronization labels in both automata.

\section{Automata chain}

In this section we introduce the concept of automata chain whose goal is to represent an automaton into pieces. The correct slicing of an automaton is defined as well as the automaton reconstruction which is the automaton obtained after the reconstruction of an automata chain. A new synchronization operation, performed on automata chains, is then presented.

Definition 4 (Automata chain) A sequence of automata $\left(A^{1}, \ldots, A^{n}\right)$ with $A^{i}=\left(Q^{i}, E^{i}, T^{i}, I^{i}, F^{i}\right)$ is called automata chain, and denoted $\mathcal{E}_{A}$, if:

$-\forall i, j, E^{i}=E^{j}$

- $\forall i, j, j>i, \forall q, q \in Q^{i} \cap Q^{j} \Rightarrow q \in F^{i} \wedge q \in I^{i+1}$, and

- $\forall i, j, \forall q, q^{\prime}$, if $\left\{q, q^{\prime}\right\} \subseteq Q^{\imath} \cap Q^{j}$ then $\forall p$, path of $A^{i}$ between $q$ and $q^{\prime}, p$ is also a path of $A^{j}$.

In the following, the superscript $i$ refers to the $i$ th automaton of the chain. An automata chain is given Figure 1. To simplify, the labels over the transitions are not represented. By definition, a state must not appear in two different automata of the chain except if it belongs to the boundaries of two successive automata, i.e the state is a final state of the former and an initial state of the later. The last item of the previous definition requires similar path between the states on the boundary of two consecutive automata (see for example the states 4 and 5 for the second and third automata of the chain). The third condition of the definition is necessary to obtain the Property 1 (defined later, see the proof in annex). 


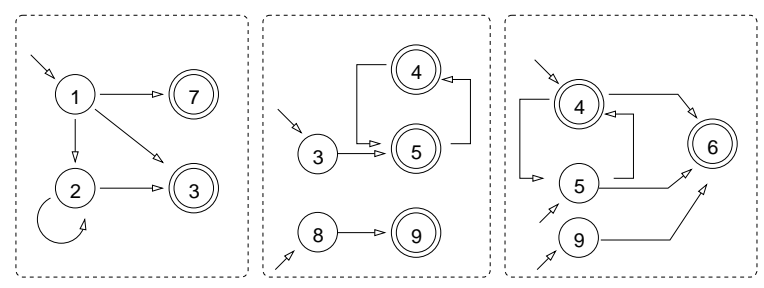

Fig. 1. Chain of three automata

Definition 5 (Trajectory reconstruction) Let $\operatorname{traj}^{i}=\left(\left(q_{0}^{i}, \ldots, q_{m i}^{i}\right)\right.$, $\left.\left(l_{1}^{i}, \ldots, l_{m i}^{i}\right)\right)$ be $n$ trajectories such that $\forall i, q_{m i}^{i}=q_{0}^{i+1}$. Then the trajectory traj resulting from the reconstruction of the $n$ trajectories traj ${ }^{i}$, is defined by: traj $=\left(\left(\begin{array}{llllll}q_{0}^{1} & \ldots\end{array} q_{m 1}^{1}, q_{1}^{2}, \ldots, q_{m 2}^{2}, \ldots, q_{1}^{n}, \ldots, q_{m n}^{n}\right)\right.$, $\left.\left(l_{1}^{1}, \ldots, l_{m 1}^{1}, l_{1}^{2}, \ldots, l_{m 2}^{2}, \ldots, l_{1}^{n}, \ldots, l_{m n}^{n}\right)\right)$.

Definition 6 (Automata chain trajectory) Let $\mathcal{E}_{A}$ be an automata chain $\left(A^{1}, \ldots, A^{n}\right)$. A trajectory of $\mathcal{E}_{A}$ is any trajectory obtained by the reconstruction of any $n$ trajectories traj ${ }^{i}$ from each of the $n$ automata $A^{i}$.

An example of trajectory on the automata chain presented Figure 1 is $((1,2,2,2,3,5,4,5,6), l)$ where $l$ is the sequence of labels of the transitions.

Definition 7 (Correct slicing) Let $A$ be an automaton and $\mathcal{E}_{A}=$ $\left(A^{1}, \ldots, A^{n}\right)$ be an automata chain. $\mathcal{E}_{A}$ is a correct slicing of $A$ iff the set of trajectories of $\mathcal{E}_{A}$ is equal to the set of trajectories of $A$. We denote $\operatorname{Sli}(A)$ a correct slicing of $A$ into an automata chain $\mathcal{E}_{A}$ such that $\mathcal{E}_{A}=\operatorname{Sli}(A)$.

The chain in Figure 1 is a correct slicing of the automaton of Figure 2.

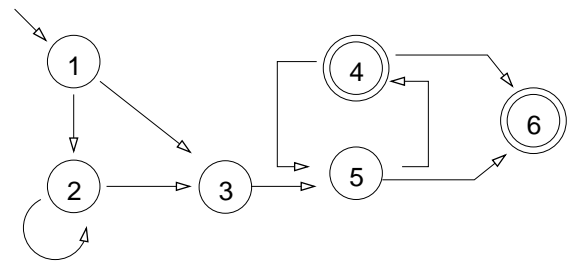

Fig. 2. The automata chain $\mathcal{E}_{A}$ presented in Figure 1 is one of the correct slicings of this automaton. It can be obtained (see Property 1) by reconstruction of $\mathcal{E}_{A}$.

The reconstruction operation builds an automaton (see Figure 2) from the sequence of automata of an automata chain (see Figure 1). In a first step, all the states and transitions are kept, the initial states being the initial states of the first automaton (state 1) and the final states being the final states of the last one (states 4 and 6 ). In a second step, the states which are not reachable from 
these new initial states (as states 8,9 ) or not leading to a final state (as state 7 ) are deleted as well as the transitions from or to these deleted states.

Definition 8 (Automaton reconstruction) Let $\mathcal{E}_{A}=\left(A^{1}, \ldots, A^{n}\right)$ be an automata chain with $A^{i}=\left(Q^{i}, E^{i}, T^{i}, I^{i}, F^{i}\right)$. We call reconstruction of the chain $\mathcal{E}_{A}$, the simplified automaton obtained from $A_{R}=\left(Q_{R}, E_{R}, T_{R}, I_{R}, F_{R}\right)$ defined as follows:

$-Q_{R}=Q^{1} \cup \ldots \cup Q^{n}$

$-E_{R}=E^{1}=\ldots=E^{n}$

$-T_{R}=T^{1} \cup \ldots \cup T^{n}$,

$-I_{R}=I^{1}$, and

$-F_{R}=F^{n}$.

Property 1 Let $A$ be an automaton and $\mathcal{E}_{A}$ an automata chain. If $\mathcal{E}_{A}$ is a correct slicing of $A$, then $A$ is obtained by the reconstruction of $\mathcal{E}_{A}$.

Proof: The proof is given in Annex.

The reconstruction of $\mathcal{E}_{A}$ is denoted $S l i^{-1}\left(\mathcal{E}_{A}\right)$. If $\mathcal{E}_{A}$ is a slicing of $A$, then $A=S l i^{-1}\left(\mathcal{E}_{A}\right)$ (Property 1 ). We now see how the size of the automata chain can be reduced by refinement without loss of information.

Definition 9 (I-refined (F-refined) automata chain) An automata chain $\mathcal{E}_{A}=\left(A^{1}, \ldots, A^{n}\right)$ with $A^{i}=\left(Q^{i}, E^{i}, T^{i}, I^{i}, F^{i}\right)$ is called I-refined (resp. Frefined) iff $\forall i, I^{i+1} \subseteq F^{i}$ (resp. $\left.F^{i} \subseteq I^{i+1}\right)$.

Definition 10 (I-Refinement) Let $\mathcal{E}_{A}=\left(A^{1}, \ldots, A^{n}\right)$ be an automata chain with $A^{i}=\left(Q^{i}, E^{i}, T^{i}, I^{i}, F^{i}\right)$. We call I-refinement of $\mathcal{E}_{A}$ a sequence $\mathcal{E}_{A^{\prime}}=$ $\left(A^{\prime 1}, \ldots, A^{\prime n}\right)$ such that $\exists q, \exists i>1, q \in I^{i} \wedge q \notin F^{i-1}$ with:

$-\forall j \neq i, A^{\prime j}=A^{j}$,

- $A^{\prime i}$ is the simplified automaton from $\left(Q^{i}, E^{i}, T^{i}, I^{i} \backslash\{q\}, F^{i}\right)$.

F-refinement can be defined in an analog way as I-refinement. We use the generic term of refinement to either I-refinement or F-refinement.

Property 2 Let $\mathcal{E}_{A}$ be an automata chain. The sequence of automata $\mathcal{E}_{A^{\prime}}$ obtained by refinement of $\mathcal{E}_{A}$ is a chain. Moreover, the refinement operation on automata chain preserves the equality of the reconstructed automata.

Proof. It is obvious that the trajectories of $\mathcal{E}_{A^{\prime}}$ are trajectories of $\mathcal{E}_{A}$. Let traj be a trajectory of $\mathcal{E}_{A}$. Then, there exists $\operatorname{traj}^{1}, \ldots, \operatorname{traj}^{n}$, trajectories such that $\operatorname{traj}^{i}=\left(\left(q_{0}^{i}, \ldots, q_{m i}^{i}\right),\left(l_{1}^{i}, \ldots, l_{m i}^{i}\right)\right)$ and $q_{m i}^{i}=q_{0}^{i+1} . \forall i, q_{0}^{i}$ is a state of $F^{i-1}$ and so, this state cannot be removed by I-refinement. $\forall i, q_{m i}^{i}$ is a state of $I^{i+1}$ and so, this state cannot be removed by F-refinement. Then, traj is a trajectory of $\mathcal{E}_{A^{\prime}}$.

A refinement enables us to get a smaller equivalent automata chain. The I-refined automata chain obtained by successive I-refinements of the automata chain Figure 1 is presented Figure 3. The refinement operation is specially useful in the incremental synchronization (presented later, Section 5). 


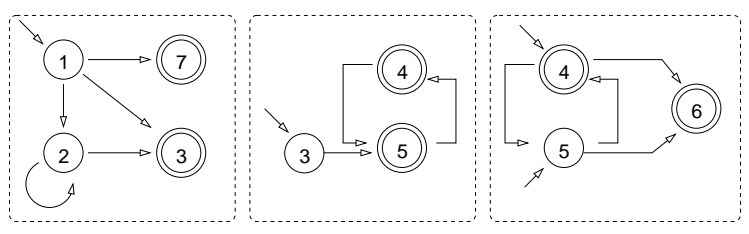

Fig. 3. I-refined automata chain $\left(I^{i+1} \subseteq F^{i}\right)$

Our interest is now the synchronization of an automata chain with an automaton.

Definition 11 (Prefix(suffix)-closed automaton) Let $A=(Q, E, T, I, F)$ be an automaton. We call prefix-closed (resp. suffix-closed) automaton of $A$, denoted $A^{+}$(resp. $\left.A^{-}\right)$, the automaton $A$ whose all states are final: $F^{+}=Q$ (resp. initial: $I^{-}=Q$ ).

We denote $A^{\#}$, the automaton which is both prefix-closed and suffix-closed $\left(A^{\#}=A^{+^{-}}=A^{-^{+}}\right)$.

Definition 12 (Automata chain synchronization) We call synchronization of an automata chain $\mathcal{E}_{A}=\left(A^{1}, \ldots, A^{n}\right)$ with an automaton $M$ the sequence denoted $\mathcal{E}_{A} \otimes M$ defined by: $\mathcal{E}_{A} \otimes M=\left(A^{1} \otimes M^{+}, A^{2} \otimes M^{\#}, \ldots, A^{n-1} \otimes\right.$ $\left.M^{\#}, A^{n} \otimes M^{-}\right)$.

When the state $q$ of $I^{i}(i \neq 1)$ is reached, the current state of $M$ is not necessarly an initial state of $M$. Thus, the synchronization uses the suffix-closure of the automaton $M$. For the same reason, the prefix-closure of $M$ is used.

Property 3 Let $\mathcal{E}_{A}$ be an automata chain and $M$ an automaton, then $\mathcal{E}_{A} \otimes M$ is an automata chain.

Proof. We denote $\mathcal{E}_{A}=\left(A^{1}, \ldots, A^{n}\right)$ with $A^{i}=\left(Q^{i}, E^{i}, T^{i}, I^{i}, F^{i}\right)$. Let $M=\left(Q_{M}, E_{M}, T_{M}, I_{M}, F_{M}\right)$. We note $\mathcal{E}_{A} \otimes M=\left(A_{\otimes}^{1}, \ldots, A_{\otimes}^{n}\right)$ with $A_{\otimes}^{i}=$ $\left(Q_{\otimes}^{i}, E_{\otimes}^{i}, T_{\otimes}^{i}, I_{\otimes}^{i}, F_{\otimes}^{i}\right)$.

- $\forall i, j, E_{\otimes}^{i}=E_{\otimes}^{j}=E \cup E_{M}$,

- $\forall i, j, j>i, \forall\left(q, q_{M}\right),\left(q, q_{M}\right) \in Q_{\otimes}^{i} \cap Q_{\otimes}^{j}$

- $q \in F^{i} \Rightarrow\left(q, q_{M}\right) \in F_{\otimes}^{i}$,

- Either $j=i+1$, and then $\left(q, q_{M}\right) \in Q_{\otimes}^{i+1}\left(\left(q, q_{M}\right)\right.$ has not been removed by the simplification). $q \in I^{i+1} \Rightarrow\left(q, q_{M}\right) \in I_{M}^{i+1}$. Or $j>i+1$ and then $q \in I^{i+1} \cap F^{i+1}$ and $\left(q, q_{M}\right) \in I_{M}^{i+1} \cap F_{M}^{i+1}$, and so, $\left(q, q_{M}\right)$ is not removed by the simplification of the automaton.

- $\forall i, j, \quad \forall\left\{\left(q_{0}, q_{M 0}\right),\left(q_{m}, q_{M m}\right)\right\} \subseteq Q_{\otimes}^{i} \cap Q_{\otimes}^{j}$. Let $p$ be a path on $A_{\otimes}^{i}$ so that $p=\left(\left(\left(q_{0}, q_{M 0}\right), \ldots,\left(q_{m}, q_{M m}\right)\right)\left(l_{1}, \ldots, l_{m}\right)\right)$. Then, $\left(\left(q_{M 0}, \ldots, q_{M m}\right), \quad\left(l_{1} \cap E_{M}, \ldots, l_{m} \cap E_{M}\right)\right) \quad$ is a path on $M$, and $\left(\left(q_{0}, \ldots, q_{m}\right),\left(l_{1} \cap E, \ldots, l_{m} \cap E\right)\right)$ is a path on $A^{i}$ and so on $A^{j}$ (since $\left.\left\{q_{0}, q_{m}\right\} \subseteq Q^{i} \cap Q^{j}\right)$. Thus, $p$ is a path on $A_{\otimes}^{j}$. 
Property 4 Let $\mathcal{E}_{A}$ be an automata chain and $M=\left(Q_{M}, E_{M}, T_{M}, I_{M}, F_{M}\right)$ then $\mathcal{E}_{A} \otimes M$ is a correct slicing of $\operatorname{Sli}^{-1}\left(\mathcal{E}_{A}\right) \otimes M$.

Proof. We use the same notation as in the previous proof. Let $\operatorname{traj}_{\otimes}=$ $\left(\left(q_{0}, \ldots, q_{m}\right),\left(l_{1}, \ldots, l_{m}\right)\right)$, a trajectory of $\operatorname{Sli}^{-1}\left(\mathcal{E}_{A}\right) \otimes M$ so that $q_{i}=\left(q_{i}^{A}, q_{i}^{M}\right)$ and $l_{i}=\Theta\left(l_{i}^{A}, l_{i}^{M},\right)$. Then $\operatorname{traj}=\left(\left(q_{0}^{A}, \ldots, q_{m}^{A}\right),\left(l_{1}^{A}, \ldots, l_{m}^{A}\right)\right)\left(\right.$ resp. $\operatorname{traj}^{M}=$ $\left.\left(\left(q_{0}^{M}, \ldots, q_{m}^{M}\right),\left(l_{1}^{M}, \ldots, l_{m}^{M}\right)\right)\right)$ is a trajectory of $\operatorname{Sli}^{-1}\left(\mathcal{E}_{A}\right)$ (resp. $\left.M\right)$. Then, $\exists f$ so that $\operatorname{traj}^{j}=\left(\left(q_{f(j)}^{A}, \ldots, q_{f(j+1)}^{A}\right),\left(l_{f(j)+1}^{A}, \ldots, l_{f(j+1)}^{A}\right)\right)$ is a trajectory of $A^{j}$ with traj is the reconstruction of the $n$ trajectories $\operatorname{traj}^{j}$. Then, $\operatorname{traj}_{\otimes}^{j}=$ $\left(\left(q_{f(j)}, \ldots, q_{f(j+1)}\right),\left(l_{f(j)+1}, \ldots, l_{f(j+1)}\right)\right)$ is a trajectory of $A^{j} \otimes M^{\#}\left(A^{1} \otimes M^{+}\right.$ for $j=1, A^{n} \otimes M^{-}$for $\left.j=n\right)$, and $t r a j_{\otimes}$ is a trajectory of $\mathcal{E}_{A} \otimes M$.

In the same way, we can prove that any trajectory of $S l i^{-1}\left(\mathcal{E}_{A} \otimes M\right)$ is a trajectory of $S l i^{-1}\left(\mathcal{E}_{A}\right) \otimes M$. Then, $\mathcal{E}_{A} \otimes M$ is a correct slicing of $S l i^{-1}\left(\mathcal{E}_{A}\right) \otimes M$.

\section{Diagnosis by slices}

This section proposes to use the formalism of automata chains to represent the observations and to compute, given Property 4, the system diagnosis. The section 5 then presents how to compute the diagnosis incrementally.

Let us first recall the definitions used in the domain of discrete-event systems diagnosis where the system is traditionally modelled by an automaton.

Definition 13 (Model) The model of the system, denoted Mod, is an automaton $\left(Q^{M o d}, E^{M o d}, T^{M o d}, I^{M o d}, F^{M o d}\right) . I^{M o d}$ is the set of possible states at $t_{0}$. All the states of the system may be final, thus $F^{\text {Mod }}=Q^{\text {Mod }}$. The set of observable events of the system is denoted $E_{O b s}^{M o d} \subseteq E^{M o d}$.

The model of the system describes its behaviour and the trajectories of Mod represent the evolutions of the system. Let us remark that we do not have any information on the final states of $M o d$, and so $\mathrm{Mod}^{+}=\operatorname{Mod}$ and $\mathrm{Mod}^{\#}=\mathrm{Mod}^{-}$.

Let us turn to observations and diagnosis definitions. We consider that the observable events are observed by sensors and sent via communication channels to an unique supervisor. Therefore, the observations are subject to uncertainties: the clocks of the sensors are not synchronized (see for instance [7]), the transfer policy and duration are variable or partially unknown, some observations may even be lost, etc. Generally, we don't know the total order on the observations emitted by the system. Consequently, the observations are represented by an automaton, each trajectory of which represents a possible order of emission of the observations.

Definition 14 (Observation automaton) The observation automaton, denoted $\mathrm{Obs}_{n}$, is an automaton describing the observations emitted by the system during the period $\left[t_{0}, t_{n}\right]$.

Definition 15 (Diagnosis) The diagnosis, denoted $\Delta_{n}$, is an automaton describing the possible trajectories on the model of the system compatible with the observations sent by the system during the period $\left[t_{0}, t_{n}\right]$. 
The diagnosis can be formally defined as resulting from the synchonization of the automaton representing the model $(M o d)$, and the automaton representing the observations $O b s_{n}$ on the period $\left[t_{0}, t_{n}\right]$. We have:

$$
\Delta_{n}=O b s_{n} \otimes \operatorname{Mod}
$$

Using Property 4, the diagnosis by slices can be computed. The idea is to compute diagnosis slices, corresponding to observations slices. The global diagnosis can be reconstructed from the diagnosis automata chain that is obtained.

Definition 16 (Diagnosis by slices - Diagnosis slice) Let Mod be the system model and $\mathrm{Obs}_{n}$ the observation automaton. Let $\mathcal{E}_{O b s_{n}}=\left(O b s^{1}, \ldots, O b s^{n}\right)$, be a correct slicing of $\mathrm{Obs}^{n}$. The synchronization (see definition 12) of $\mathcal{E}_{\mathrm{Obs}_{n}}$ with $\mathrm{Mod}$, i.e $\mathcal{E}_{\mathrm{Obs}_{n}} \otimes \mathrm{Mod}=\left(\mathrm{Obs}^{1} \otimes \mathrm{Mod}, \mathrm{Obs}^{2} \otimes \mathrm{Mod}^{\#}, \ldots, \mathrm{Obs}^{n} \otimes \mathrm{Mod}^{\#}\right)$ is the diagnosis by slices of the system.

It can be denoted by the diagnosis automata chain $\left(\Delta^{1}, \ldots, \Delta^{n}\right)$, where $\Delta^{i}$ is called the ith diagnosis slice of the system.

Using Property 4, it can be proved that the diagnosis by slices of a system, here $\mathcal{E}_{\mathrm{Obs}_{n}} \otimes \operatorname{Mod}$, correctly represents the diagnosis computed on the global observations since the reconstruction of $\mathcal{E}_{\mathrm{Obs}_{n}} \otimes \operatorname{Mod}$ equals the global diagnosis:

Result $1 \Delta_{n}=S l i^{-1}\left(\mathcal{E}_{O b s_{n}} \otimes M o d\right)$

\section{Incremental diagnosis}

In the diagnosis by slices as presented above, the $i$ th diagnosis slice, $\Delta^{i}$, is computed independently from the others, by synchronizing the $i$ th observation slice from the chain $\mathcal{E}_{O b s_{n}}, \mathrm{Obs}^{i}$, with the system model $\mathrm{Mod}^{\#}$. One of the interests of the observation slicing is to make the parallelized computation of each diagnosis slice possible. In this section, we focus on another approach, which elaborates an incremental diagnosis, using $\Delta^{i-1}$ to restrict the set of initial states of Mod when computing $\Delta^{i}{ }^{3}$. In this section we first present a new definition of the synchronization for the incremental case and tackle the specific problem of incremental diagnosis.

Definition 17 (Restriction) Let $A=(Q, E, T, I, F)$ be an automaton. The automata restriction of $A$ by the states of $I^{\prime}$, denoted $A\left[I^{\prime}\right]$, is the automaton $A^{\prime}=\left(Q, E, T, I \cap I^{\prime}, F\right)$.

In the incremental synchronization the set of initial states of an automaton of the chain is restricted by the set of final states of its predecessor.

Definition 18 (Incremental synchronization) The incremental synchronization of the automata chain $\mathcal{E}_{A}=\left(A^{1}, \ldots, A^{n}\right)$ with the automaton $M$, denoted $\mathcal{E}_{A} \odot M$ is defined as $\left(A^{\prime 1}, \ldots, A^{\prime n}\right)$ with $A^{\prime i}=\left(Q^{\prime i}, E^{\prime}, T^{\prime i}, I^{\prime i}, F^{\prime i}\right)$ and:

\footnotetext{
${ }^{3}$ We could conversely use $\Delta^{i}$ to restrict the set of final states of $M o d$ when computing the diagnosis $\Delta^{i-1}$.
} 


$$
\begin{aligned}
& -A^{1}=A^{1} \otimes M^{+} \\
& -\forall i \in\{2, \ldots, n-1\}, A^{\prime i}=\left(A^{i} \otimes M^{\#}\right)\left[F^{\prime i-1}\right] \text { and } \\
& -A^{\prime n}=\left(A^{n} \otimes M^{-}\right)\left[F^{\prime n-1}\right] .
\end{aligned}
$$

Property 5 Let $\mathcal{E}_{A}$ be an automata chain and $M$ an automaton. Then $\mathcal{E}_{A} \odot M$ is the automata chain obtained by successive I-refinements of $\mathcal{E}_{A} \otimes M$.

Proof. It is clear that the chain of automata $\mathcal{E}_{A} \odot M$ can be obtained by successive I-refinements of $\mathcal{E}_{A} \otimes M$ since the definition is identical except the removal of initial states $q_{i}$ of the $i$ th automaton not in the set of final states of the $(i-1)$ th automaton. It is also clear that $\mathcal{E}_{A} \odot M$ is I-refined since we have $\forall i, I^{i} \subseteq F^{i-1}$.

Property 6 Let $\mathcal{E}_{A}$ be an automata chain and $M=\left(Q_{M}, E_{M}, T_{M}, I_{M}, F_{M}\right)$ an automaton. We have $\operatorname{Sli}^{-1}\left(\mathcal{E}_{A} \odot M\right)=\operatorname{Sli}^{-1}\left(\mathcal{E}_{A} \otimes M\right)$.

This can be proved using Property 2 and Property 5 .

Given this new definition of synchronization, a formalization of incremental diagnosis can be proposed. Provided that $\mathcal{E}_{O b s_{n}}=\left(O b s^{1}, \ldots, O b s^{n}\right)$ is a correct slicing of $O b s_{n}$ we have: $\Delta_{n}=O b s_{n} \otimes \operatorname{Mod}=\operatorname{Sli}^{-1}\left(\mathcal{E}_{O b s_{n}} \odot \mathrm{Mod}\right)$.

We note $\forall i, \mathcal{E}_{\mathrm{Obs}_{i}}=\left(O b s^{1}, \ldots, O b s^{i}\right)$, the automata chain of the first $i$ observations automata. Let $i<n$, and $\mathcal{E}_{\Delta_{i}}=\left(\Delta^{1}, \ldots, \Delta^{i}\right)$ the automata chain resulting from the incremental synchronization of $\mathcal{E}_{\mathrm{Obs}_{i}}$ with the system model Mod. We can incrementally compute $\mathcal{E}_{\Delta_{i+1}}=\mathcal{E}_{\mathrm{Obs}_{i+1}} \odot \operatorname{Mod}$ as follows:

Result $2 \mathcal{E}_{\Delta_{i+1}}=\left(\Delta^{1}, \ldots, \Delta^{i}, \Delta^{i+1}\right)$ with $\Delta^{i+1}=\left(\operatorname{Obs}^{i+1} \otimes \operatorname{Mod}^{\#}\right)\left[F_{\Delta}^{i}\right]$ where $F_{\Delta}^{i}$ is the set of final states of $\Delta^{i}$.

This result comes from the fact that $\operatorname{Mod}^{-}=\operatorname{Mod}^{\#}$ (all the states in Mod are final states). Thus it is possible to compute the automata chain that represents the diagnosis in an incremental way by synchronizing the one after the other each of the automata of the observation chain.

We note $\mathrm{Obs}_{i}$ the reconstruction of $\mathcal{E}_{\mathrm{Obs}_{i}}$. Then:

Result 3 Let $\Delta_{i}=S \operatorname{Si}^{-1}\left(\mathcal{E}_{\Delta_{i}}\right)$. Then, $\Delta_{i}=O b s_{i} \otimes \operatorname{Mod}$.

\section{Temporal windows diagnosis}

It has been proved above that, at the condition to have a correct slicing of the observation automaton, it is possible to incrementally compute the global system diagnosis by considering in sequence the slices of observations and computing for each of them its diagnosis slice. In this section, we show that this result can be instantiated to the case where the observation automaton is sliced according to time, according to temporal windows. Firstly, we extend the definition of correct slicing to temporally correct slicing by requiring temporal properties. Then, the incremental computation is demonstrated as valid on temporal windows which correctly slice the observation automaton. 
Definition 19 (Correct sequence of temporal windows) Let $t_{i}$ be time instants and $\left[t_{0}, t_{n}\right]$ be the global diagnosis temporal window. A sequence of temporal windows is correct w.r.t $\left[t_{0}, t_{n}\right]$ iff it is a sequence $\mathcal{W}=$ $\left(\mathcal{W}_{1}, \ldots, \mathcal{W}_{i}, \ldots, \mathcal{W}_{n}\right)$ such that $\forall i \in\{1, \ldots, n\}, \mathcal{W}_{i}=\left[t_{i-1}, t_{i}\right]$

Definition 20 (Temporally correct slicing) Let $O b s_{n}$ be the observation automaton on $\left[t_{0}, t_{n}\right]$. The automata chain $\mathcal{E}_{O b s_{n}}=\left(\mathrm{Obs}^{\mathcal{W}_{1}}, \ldots, \mathrm{Obs}^{\mathcal{W}_{n}}\right)$ is a temporally correct slicing of $\mathrm{Obs}_{n}$ according to $\mathcal{W}=\left(\mathcal{W}_{1}, \ldots, \mathcal{W}_{i}, \ldots, \mathcal{W}_{n}\right)$ iff:

- the slicing is correct;

- $\mathcal{W}$ is a correct sequence of temporal windows w.r.t $\left[t_{0}, t_{n}\right]$; and

- for each trajectory in $\mathrm{Obs}^{\mathcal{W}_{i}}$, the transitions have occured during $\left[t_{i-1}, t_{i}\right]$ (i.e the observations labelling the transitions have been emitted by the system in $\left.\mathcal{W}_{i}\right)$.

Let us remark that, for any $i \in\{1, \ldots, n\}$, the initial states of $O b s^{\mathcal{W}_{i}}$ are possible states at $t_{i-1}$ and that the final states of $O b s^{\mathcal{W}_{i}}$ are possible states at $t_{i}$. Note also that, if a final state of a temporal window can be reached by two trajectories, it is required that both trajectories have occured during the temporal window, i.e the final state is a possible state in $t_{i}$ whatever the trajectory used to get it.

The results of section 4 can be used in the case of temporally correct slicing. Let us denote $\forall i, \mathcal{E}_{O b s_{\mathcal{W}_{i}}}=\left(O b s^{\mathcal{W}_{1}}, \ldots, O b s^{\mathcal{W}_{i}}\right)$. Let $i<n$, and

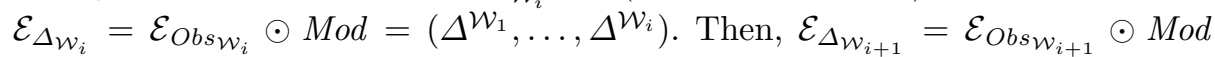
can be computed as follows:

Result $4 \mathcal{E}_{\Delta_{\mathcal{W}_{i+1}}}=\left(\Delta^{\mathcal{W}_{1}}, \ldots, \Delta^{\mathcal{W}_{i}}, \Delta^{\mathcal{W}_{i+1}}\right)$ with $\Delta^{\mathcal{W}_{i+1}}=\left(O b s^{\mathcal{W}_{i+1}} \otimes\right.$ $\left.\operatorname{Mod}^{\#}\right)\left[F_{\Delta}^{\mathcal{W}_{i}}\right]$ where $F_{\Delta}^{\mathcal{W}_{i}}$ is the set of final states of $\Delta^{\mathcal{W}_{i}}$.

Let $O b s_{\mathcal{W}_{i}}$, the automaton provided by the reconstruction operation on $\mathcal{E}_{\mathrm{Obs}_{\mathcal{W}_{i}} .} . \mathrm{Obs}_{\mathcal{W}_{i}}$ represents the observations emitted on the period $\left[t_{0}, t_{i}\right]$.

Result 5 Let $\Delta_{\mathcal{W}_{i}}=S l i^{-1}\left(\mathcal{E}_{\Delta_{\mathcal{W}_{i}}}\right)$. Then, $\Delta_{\mathcal{W}_{i}}=O b s_{\mathcal{W}_{i}} \otimes$ Mod is the diagnosis of the period $\left[t_{0}, t_{i}\right]$.

The incremental computation of diagnosis from temporal windows seems promising firstly because the diagnosis gives then the possible states of the system at time $t_{i}$ w.r.t the (possibly uncertain) observations gathered at time $t_{i}$. Another good reason appears when turning into an on-line diagnosis context. The observation automata chain has now to be built on-line, i.e without knowing by advance the whole set of observations gathered on the global diagnosis window. This point will not be examined in this paper but it can be shown that taking advantage of temporal information, it is easier, on-line, to build temporally correct slicing than only correct slicing. Observations, which should be considered as possible in the general case, can be discarded as not satisfying the temporal constraints collected on the system behaviour (as delays between observations emission and reception; communication channels politics...). 


\section{Conclusion}

In this paper, we formalized the incremental computation of diagnosis for discrete-event systems. We introduced and defined the concept of automata chain that enables us to handle slices of observations and slices of diagnosis rather than global observations and global diagnosis. We proved that the diagnosis can be computed, by using automata chain, in a modular way and, more efficiently, in an incremental way. We then presented how the results can be extended to the case where observations are sliced according to temporal windows.

In the diagnostic literature the notion of incremental diagnosis is relatively new. It can be explained by the fact that, in most cases, observations are supposed to be totally ordered, received without delays, and without any loss. In these cases, the problem of slicing the observations does not exist. In [2] however, the authors examine the case where observations are uncertain and represented by partially ordered graphs. In the case of decentralized systems, Pencolé et al. [7] consider the incremental diagnosis computation applied to the on-line diagnosis for telecommunication networks. The property of safe window is defined and algorithms are given in the case where the temporal windows satisfy this property. Extensions to more complicated cases are proposed. Compared to this, our proposal is more general and give a formal view of the problem which allows to better situate the algorithmic approach proposed in [7]. In [8] an incremental approach of diagnosis is considered from a model-checking point of view.

Our study exhibits the (non trivial) correctness properties that the observation slicing, in an automata chain, has to satisfy in order to guarantee the completeness of the diagnosis computation. This first step is then essential before considering the incrementality of on-line diagnosis computation.

The next step will consider the building of the observations automata chain in the context of off-line and then on-line diagnosis. The case of on-line diagnosis is particularly interesting since the goal is to dynamically build an automata chain without having all the observations. As seen at the end of section 6 , this task can take profit of temporal information known on the system, even if, for complexity reasons, these temporal constraints are not encoded in the system model. Another interesting point is to use the concept of automata chains for the diagnosis of reconfigurable systems.

\section{Annex}

Proof (Property 1).

Let $A=(Q, E, T, I, F)$ be an automaton and $\mathcal{E}_{A}=\left(A^{1}, \ldots, A^{n}\right)$ an automata chain with $A^{i}=\left(Q^{i}, E^{i}, T^{i}, I^{i}, F^{i}\right)$ so that $\mathcal{E}_{A}$ is a correct slicing of $A$. Let $A_{R}=\left(Q_{R}, E_{R}, T_{R}, I_{R}, F_{R}\right)$ be the reconstruction of $\mathcal{E}_{A}$. We have to prove that the set of trajectories of $A$ (which is the same as the set of trajectories of $\mathcal{E}_{A}$ ) equals the set of trajectories of $A_{R}$.

Let $\mathcal{E}_{A_{1,2}}=\left(A^{1}, A^{2}\right) \cdot \mathcal{E}_{A_{1,2}}$ is an automata chain. Let $A_{1,2}$ be the reconstruction of $\mathcal{E}_{A_{1,2}}$. Let us consider a transition $\left(q, l, q^{\prime}\right)$ of $A_{1,2}$.

Remark 1: $\left\{q, q^{\prime}\right\} \subseteq Q^{1}$ or $\left\{q, q^{\prime}\right\} \subseteq Q^{2}$ (because $\left.\left(q, l, q^{\prime}\right) \in T_{1,2}=T^{1} \cup T^{2}\right)$. Consequently, if a state does not belong to $Q^{2}$ (resp. $Q^{1}$ ), it belongs to $Q^{1}$ (resp. 
$\left.Q^{2}\right)$ and its predecessor too. Moreover, if a path on $A_{1,2}$ goes from a state from $Q^{1}$ to a state from $Q^{2}$, there exists at least one state on the path belonging to $Q^{1} \cap Q^{2}$.

Remark 2: $\forall j \in\{1,2\},\left\{q, q^{\prime}\right\} \subseteq Q^{j} \Rightarrow\left(q, l, q^{\prime}\right) \in T^{j}$.

i) $\forall \operatorname{traj}=\left(\left(q_{0}, \ldots, q_{m}\right),\left(l_{1}, \ldots, l_{m}\right)\right)$, trajectory of $\mathcal{E}_{A_{1,2}}$, then traj is also a trajectory of $A_{1,2}$ since (by definition) any transition of $A^{1}$ or $A^{2}$ is a transition of $A_{1,2}, q_{0} \in I^{1}$ and $q_{m} \in F^{2}$.

ii) $\forall \operatorname{traj}=\left(\left(q_{0}, \ldots, q_{m}\right),\left(l_{1}, \ldots, l_{m}\right)\right)$, trajectory of $A_{1,2}$. Let $k$ be the smallest value in $\{0, \ldots, m\}$ so that $q_{k} \in Q^{1} \cap Q^{2}$ ( $k$ exists due to Remark 1$)$.

$\forall i \leq k, q_{i} \in Q^{1}$, so $\operatorname{traj}^{1}=\left(\left(q_{0}, \ldots, q_{k}\right),\left(l_{1}, \ldots, l_{k}\right)\right)$ is a trajectory of $A^{1}$ (cf. Remark 2).

Let us now prove that $\forall i>k, q_{i} \in Q^{2}$. Let us suppose it exists $j$, the smallest value so that $j>k$ and $q_{j} \notin Q^{2} . q_{j} \in Q^{1}$ and, due to Remark $1, q_{j-1} \in Q^{1} \cap Q^{2}$. For the same reason as for $k, \exists l$ the smallest value so that $l>j$ and $q_{l} \in Q^{1} \cap Q^{2}$. The path $p=\left(\left(q_{j-1}, \ldots, q_{l}\right),\left(l_{j}, \ldots, l_{l}\right)\right)$ is a path of $A^{1}$. But, since $q_{j-1}$ and $q_{l}$ are both belonging to $Q^{1} \cap Q^{2}, p$ is also a path of $A^{2}$. It implies that $q_{j}$ is a state of $Q^{2}$, which is in contradiction with the existence of $j$. So, $\forall i>k, q_{i} \in Q^{2}$. And $\operatorname{traj}^{2}=\left(\left(q_{k}, \ldots, q_{m}\right),\left(l_{k+1}, \ldots, l_{m}\right)\right)$ is a trajectory of $A^{2} . t r a j$ is built by reconstruction of $\operatorname{traj}^{1}$ and $t r a j^{2}$. It is then a trajectory of $\mathcal{E}_{A_{1,2}}$.

Since the trajectories of $A_{1,2}$ and $\left(A^{1}, A^{2}\right)$ are equal, $\mathcal{E}_{A}$ and $\left(A_{1,2}, A^{3}, \ldots, A^{n}\right)$ have the same trajectories. We define recursively $\forall i>2, A_{1, i}$ the reconstruction of $\left(A_{1, i-1}, A^{i}\right)$. Then, we prove recursively that $\mathcal{E}_{A}$ has the same trajectories as $\left(A_{1, i}, A^{i+1}, \ldots, A^{n}\right)$ in particular $\left(A_{1, n}\right)=\left(A_{R}\right)$. So, $\mathcal{E}_{A}$ and $A_{R}$ have the same trajectories. As $\mathcal{E}_{A}$ is a correct slicing of $A, A=A_{R}$.

\section{References}

1. Cassandras, C.G., Lafortune, S.: Introduction to Discrete Event Systems. Kluwer Academic Publishers (1999)

2. Baroni, P., Lamperti, G., Pogliano, P., Zanella, M.: Diagnosis of large active systems. Artificial Intelligence 110 (1999) 135-183

3. Cordier, M.O., Thiébaux, S.: Event-based diagnosis for evolutive systems. In: 5th International Workshop on Principles of Diagnosis (DX-94). (1994) 64-69

4. Barral, C., McIlraith, S., Son, T.: Formulating diagnostic problem solving using an action language with narratives and sensing. In: International Conference on Knowledge Representation and Reasoning (KR'2000). (2000) 311-322

5. Console, L., Picardi, C., Ribaudo, M.: Diagnosis and diagnosability analysis using PEPA. In: 14th European Conference on Artificial Intelligence (ECAI-2000), Berlin, Allemagne (2000) 131-135

6. Lunze, J.: Discrete-event modelling and diagnosis of quantized dynamical systems. In: 10th International Workshop on Principles of Diagnosis (DX-99), Loch Awe, Écosse, Royaume Uni (1999) 147-154

7. Pencolé, Y., Cordier, M.O., Rozé, L.: Incremental decentralized diagnosis approach for the supervision of a telecommunication network. In: 12th International Workshop on Principles of Diagnosis (DX'01). (2001) 151-158

8. Cordier, M.O., Largouët, C.: Using model-checking techniques for diagnosing discrete-event systems. In: 12th International Workshop on Principles of Diagnosis (DX'01). (2001) 39-46 